\title{
Accroissement du taux d'acide linoléique dans le lait de vache
}

\author{
par \\ Simonne KUZDZAL-SAVOIE, W. KUZDZAL \\ et M. ILTCHENKO* \\ Station Centrale de Recherches Laitières et de Technologie \\ des Produits Animaux, I.N.R.A., C.N.R.Z., \\ 78350 Jouy-en-Josas
}

\section{I. - INTRODUCTION}

La matière grasse du lait de vache contient normalement 1,0-1,5 p. 100 d'acide linoléique, 0,4-0,8 p. 100 d'acide linolénique et 0,3-0,4 p. 100 d'acide arachidonique (cf. Kuzdzal-Savoie et Kuzdzal, 1961).

Certains nutritionnistes ont attiré l'attention sur la faible teneur du lait de vache en acides essentiels et particulièrement en acide linoléique. Selon Holman (cf. Holman, 1968 ; Soderhjelm et al., 1970) l'exigence minimale en acide linoléique correspondrait pour l'homme à 1 p. 100 des calories totales de la ration ; le taux optimal se situant à 4 p. 100 . Si le risque de déficience en acides essentiels est minime ou même inexistant lorsque l'alimentation est diversifiée, ce risque pourrait cependant exister - en se basant sur les résultats des travaux de Holman et al. - , lorsque le lait de vache constitue le seul aliment distribué aux enfants comme cela est souvent le cas dans l'alimentation des nouveau-nés.

Des tentatives ayant pour objectif l'accroissement du taux des acides essentiels dans le lait de vache et plus spécialement l'accroissement du taux d'acide linoléique (cis,cis-octadéca-9,12-diénoïque), acide indispensable (Le Breton et Ferret, 1959), ont donc été entreprises.

Les essais effectués par divers auteurs (cf Story et al., 1967) ou par nous-mêmes (Kuzdzal-Savoie, 1964) et consistant à enrichir en matière grasse poly-insaturée la ration alimentaire des vaches lai-

\footnotetext{
* Stagiaire du Gouvernement Français de décembre 1972 à avril 1973.

Adresse actuelle : pl. Mickiewicza 9 KV6 - 290000 Lvôv - Centre (U.R.S.S.).
} 
tières se sont révélés peu efficaces. On a observé, par exemple, un accroissement du taux d'acide linoléique de 1,2 p. 100 à 2,4 p. 100 à la suite d'une distribution journalière de $1,5 \mathrm{~kg}$ de tourteau gras de soja (à 20 p. 100 de matière grasse) en supplément alimentaire.

L'existence au niveau du rumen du phénomène de biohydrogénation s'oppose en effet à tout accroissement notable du taux des acides essentiels dans le lait.

Afin de " courcicuiter " l'action hydrogénante des micro-organismes du rumen, Scott et al. (1970) ont proposé d'introduire dans la ration des vaches laitières un supplément alimentaire constitué par une poudre "Spray » obtenue à partir d'une émulsion d'huile de carthame dans une solution aqueuse de caséinate de sodium, traitée à la formaldéhyde.

Plowman et al. (1971) utilisèrent le procédé proposé par Scott et al. (1970) et obtinrent, avec une distribution journalière de $1,5 \mathrm{~kg}$ de poudre (contenant 60 p. 100 d'huile de carthame), 30 à 35 p. 100 d'acide linoléique dans la matière grasse du lait.

Kristensen et al. (1974), au Danemark utilisèrent de l'huile de soja "protégée " et obtinrent 12,4 p. 100 d'acide linoléique dans le lait pour une distribution journalière de l'ordre de $1 \mathrm{~kg}$ d'aliment spécial représentant environ $500 \mathrm{~g}$ d'huile de soja à $53 \mathrm{p} .100$ d'acide linoléique.

Notre objectif était d'obtenir dans la matière grasse du lait de vache un taux d'acide linoléique voisin du taux de ce même acide dans le lait de femme, c'est-à-dire 10 p. 100 environ des acides gras totaux.

\section{II. - PARTIE EXPERIMENTALE}

L'aliment spécial fut préparé par le Laboratoire de Technologie Laitière à Rennes (Institut National de la Recherche Agronomique). $112 \mathrm{~kg}$ d'huile de tournesol (Aurea) contenant 62 p. 100 d'acide linoléique et additionnés de 0,5 g d'acétate DL- $\alpha$-tocophérol par $\mathrm{kg}$ d'huile, furent incorporés à $750 \mathrm{~kg}$ de lait écrémé concentré à $54 \mathrm{p}$. 100. Après atomisation on obtint $460 \mathrm{~kg}$ de poudre à $25 \mathrm{p} .100$ de matière grasse. Cette poudre fut ensuite répartie en deux fractions équivalentes. La première fraction (aliment non traité) fut seulement agglomérée en petits cylindres de $2 \mathrm{~cm}$ de long et de $0,5 \mathrm{~cm}$ de diamètre. La seconde fraction fut soumise à un traitement de tannage. Ce procédé breveté par l'Institut National de la Recherche Agronomique dans plusieurs pays dont l'U.R.S.S., consiste en une application de produit tannant, qui provoque un durcissement de la matière protidique enrobant la poudre et protège ainsi la matière grasse incluse contre toute attaque microbienne (cf. Leroy et al., 1964 ; Zelter et al., 1970). 
Trois groupes de deux vaches ont été constitués à partir du troupeau de Bressonvilliers (Institut National de la Recherche Agronomique) : groupe $\mathrm{T}$ (témoin), groupe $\mathrm{A}$ et groupe $\mathrm{E}$.

Les vaches, de race Française Frisonne Pie Noire, produisaient chaque jour 20 à $22 \mathrm{~kg}$ de lait au début de l'expérimentation et 12 à $15 \mathrm{~kg}$ à la fin de l'expérimentation. Les vaches bénéficiaient du régime de stabulation libre. Leur ration moyenne de base était constituée par $10 \mathrm{~kg}$ de maïs déshydraté (plante entière), 3,5 kg de foin de luzerne, 2,6 kg de concentré (à base de céréales), $1 \mathrm{~kg}$ de tourteau de soja (délipidé) et $0,2 \mathrm{~kg}$ de condiment minéral. Ces aliments étaient distribués à l'auge, à l'exception du concentré donné en salle de traite. L'aliment spécial, traité ou non traité fut distribué en remplacement d'une quantité équivalente de concentré.

L'aliment spécial fut distribué à raison de $1,2 \mathrm{~kg}$ par jour pendant 1 semaine, à raison de $2,4 \mathrm{~kg}$ pendant 2 semaines et à raison de $1,2 \mathrm{~kg}$ pendant la $4^{\mathrm{e}}$ semaine. Les vaches du groupe A reçurent l'aliment non traité au cours d'une première période de 4 semaines et l'aliment traité au cours d'une seconde période de 4 semaines séparée de la première période par 1 semaine sans distribution d'aliment spécial. Les vaches du groupe E reçurent l'aliment traité (huile protégée) au cours de la première période et l'aliment non traité (huile non protégée) au cours de la deuxième période. A la fin de cette deuxième période et seulement pour les vaches du groupe $\mathrm{E}$, la quantité d'aliment non traité fut réduite un peu plus tôt que prévu : le 19 avril, les vaches du groupe $\mathrm{E}$ reçurent $1,6 \mathrm{~kg}$ d'aliment non traité au lieu de $2,4 \mathrm{~kg}$, le 20 avril $0,8 \mathrm{~kg}$ et du 20 au 28 avril $0,5 \mathrm{~kg}$ par jour.

Des prélèvements de lait furent effectués deux fois par semaine sur la traite du matin (mardi et jeudi), de février à mai 1973. A partir du lait produit par les vaches appartenant à un même groupe, un lait de mélange pondéré en fonction de la production fut constitué. Au total 25 prélèvements de lait furent effectués. Sur chaque lait de mélange on détermina le taux butyreux. Les lipides furent extraits par la méthode Röse Gottlieb, les esters méthyliques obtenus par transestérification alcaline des lipides totaux furent analysés par chromatographie en phase gazeuse, la phase stationnaire étant constituée par du succinate de butane diol imprégnant au taux de 10 p. 100 du chromosorb W.

\section{III. - RESULTATS}

Bien que la matière grasse produite à la traite du matin fut connue, le nombre très restreint des prélèvements ne permit pas d'établir de manière certaine la faible élévation de la production de matière grasse entrevue pendant la période de distribution de l'aliment spécial, notamment pour le groupe E. 
La composition de la matière grasse du lait fut par contre étudiée de manière approfondie, et les modifications des proportions relatives des acides gras constituent l'essentiel des résultats.

La variation atteignant le taux d'acide linoléique apparaît sur la figure 1. La distinction est nette entre les deux périodes pour chacun des groupes. Lorsque la matière grasse de l'aliment est "protégée " on observe un net accroissement du taux d'acide linoléique dans le lait : le taux de 12 p. 100 des acides totaux est atteint. Par contre lorsque l'huile de tournesol n'est pas protégée, le taux d'acide linoléique dans le lait ne s'élève que très modérément : de 2 à 4 p. 100 des acides totaux environ.

L'effet de la distribution du supplément alimentaire sur le taux d'acide oléique est un peu moins net (fig. 2). Si pour le groupe A on constate une nette différenciation entre les deux périodes, avec un fort accroissement d'acide oléique lorsque le groupe reçoit l'aliment non traité et un très faible accroissement lorsque le groupe reçoit l'aliment traité, pour le groupe $\mathrm{E}$, qui reçoit l'aliment traité au cours de la première période, une certaine hydrogénation doit alors survenir. Celle-ci en effet entraîne une partielle augmentation du taux d'acide oléique au cours de la première période ; cependant l'augmentation du taux d'acide oléique est beaucoup plus prononcée au cours de la seconde période (distribution de l'aliment non traité). Un taux d'acide oléique de 41 p. 100 des acides totaux est atteint.

Le taux d'acide palmitique s'abaisse généralement lorsque le taux global des acides à 18 atomes de carbone s'accroît. On observe ainsi (fig. 3) dans tous les cas, une diminution du taux d'acide palmitique ; cette diminution est cependant plus accentuée lorsque le taux d'acide oléique est particulièrement accru (groupe E, deuxième période) ou lorsque l'accroissement du taux d'acide linoléique s'accompagne d'un accroissement notable du taux d'acide oléique (groupe E, première période). Pour le groupe A, pendant la deuxième période, la diminution du taux d'acide palmitique est moindre, car elle ne doit compenser que l'augmentation du taux de l'acide linoléique et celle relativement limitée du taux de l'acide oléique ou du taux de l'acide stéarique.

Le taux de l'acide stéarique s'accroît d'une manière irrégulière (fig. 4). Le parallélisme avec les variations du taux d'acide oléique est très imparfait et le rapport acide oléique / acide stéarique varie fortement.

Des observations peuvent être faites en ce qui concerne les acides poly-insaturés mineurs. Il a été antérieurement établi (KuzdzalSavoie et Langlois, 1969) que l'acide diène conjugué formé au cours de l'hydrogénation dans le rumen occupe sur les chromatogrammes un emplacement défini : adjacent et postérieur au pic de l'acide linolénique (la phase stationnaire étant du succinate de butane diol). 
Ac. linol.

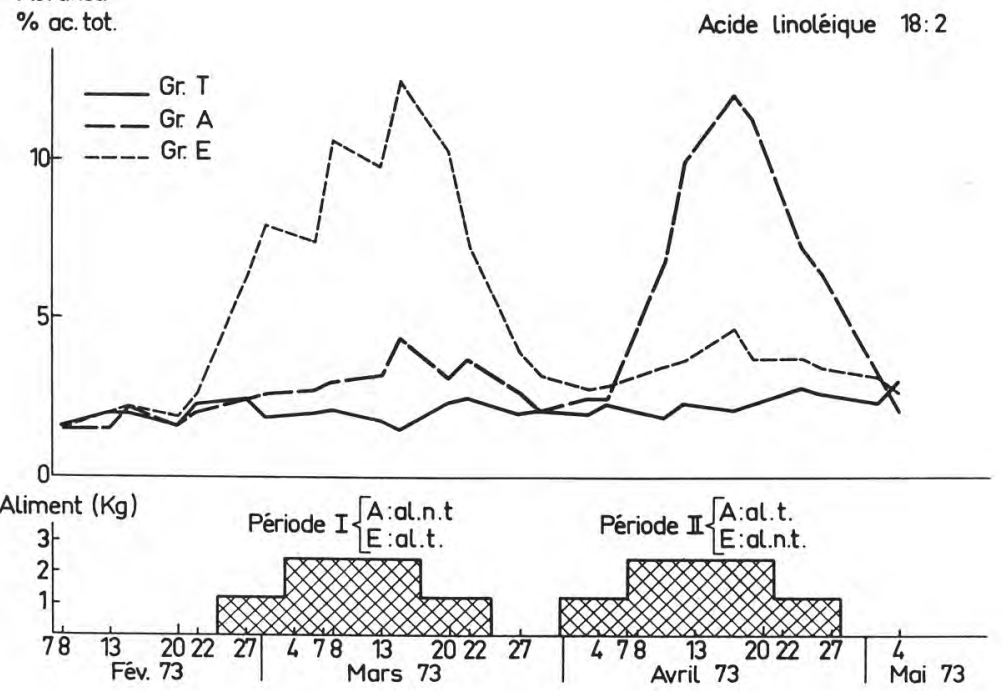

fig. 1

Variation de la proportion d'acide linoléique dans la matière grasse du lait au cours de l'expérimentation.

Les vaches du groupe A reçoivent l'aliment non traité au cours de la première période, et l'aliment traité au cours de la deuxième période. Les vaches du groupe $\mathrm{E}$ reçoivent l'aliment traité au cours de la première période et l'aliment non traité au cours de la seconde période.
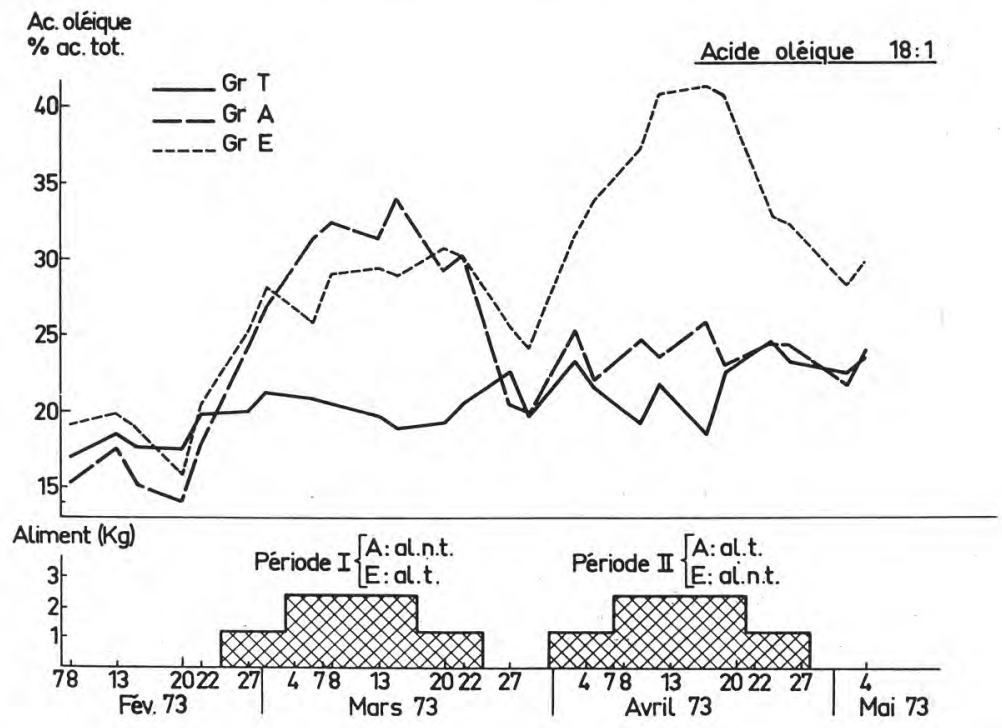

fig. 2

Variation de la proportion d'acide oléique dans la matière grasse du lait au cours de l'expérimentation. 
Ac. polm.

$\%$ oc. tot.

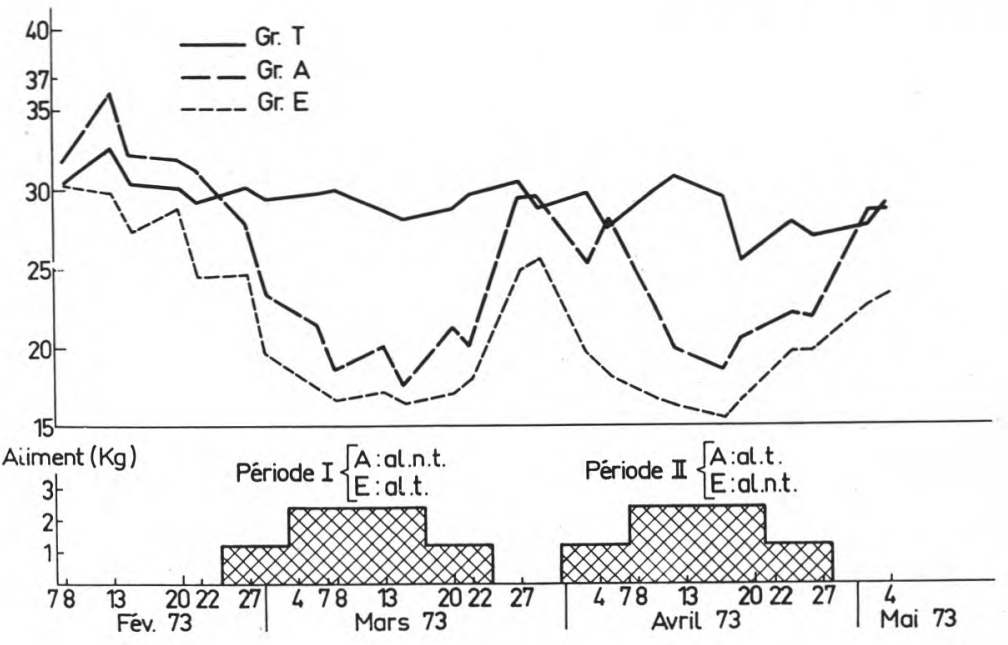

fig. 3

Variation de la proportion d'acide palmitique dans la matière grasse du lait au cours de l'expérimentation.

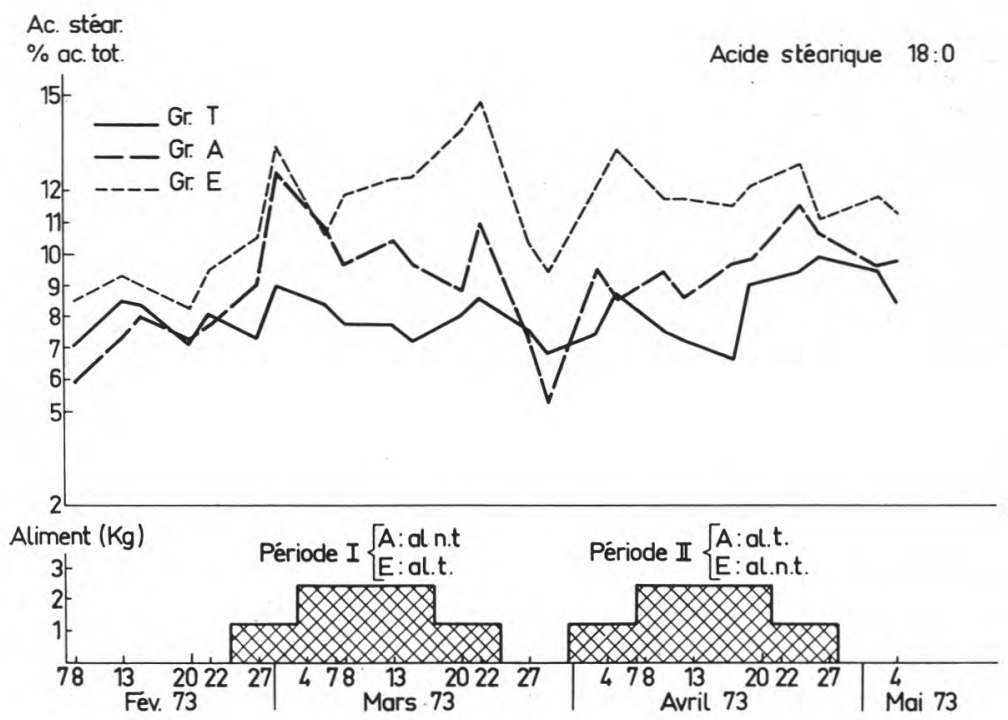

fig. 4

Variation de la proportion d'acide stéarique dans la matière grasse du lait au cours de l'expérimentation. 
La figure 5 illustre les variations du taux d'acide conjugué octadécadiénoïque dans la matière grasse du lait. Ce taux est élevé quand l'hydrogénation est active, c'est-à-dire quand l'aliment non traité est distribué aux vaches laitières (groupe A : première période ; groupe $\mathrm{E}$ : seconde période).

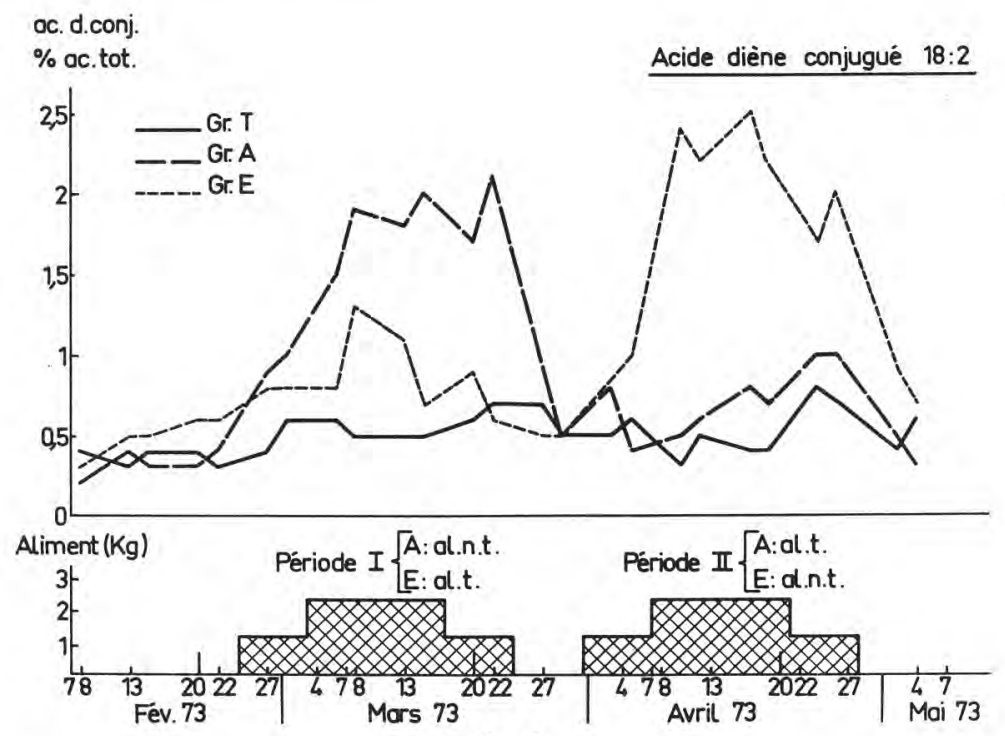

fig. 5

Variation de la proportion d'acide conjugué octadécadiènoïque dans la matière grasse du lait au cours de l'expérimentation.

A importance égale du pic de l'acide stéarique, on constate, dans le cas d'une consommation d'aliment traité, la présence d'un taux relativement faible d'acide diène conjugué accompagnant un taux d'acide linoléique fort, et au contraire dans le cas d'une consommation d'aliment non traité, un taux d'acide diène conjugué relativement élevé comparativement au taux d'acide linoléique (fig. 6).

Ainsi la variation du taux de l'acide conjugué octadécadiénoïque suit approximativement la variation de l'acide oléique, et est inverse de celle de l'acide linoléique.

$\mathrm{Au}$ fur et à mesure que s'accroît le taux d'acide linoléique dans le lait (conséquence d'une consommation d'aliment traité) le rapport acide conjugué octadécadiénoïque / acide linoléique diminue (fig. 7).

Ce rapport constitue un critère ou une mesure de l'importance de l'hydrogénation.

Les taux des acides mineurs ramifiés (iso 13, iso et antéiso 15, iso et antéiso 17) de même d'ailleurs que les taux des acides mineurs 

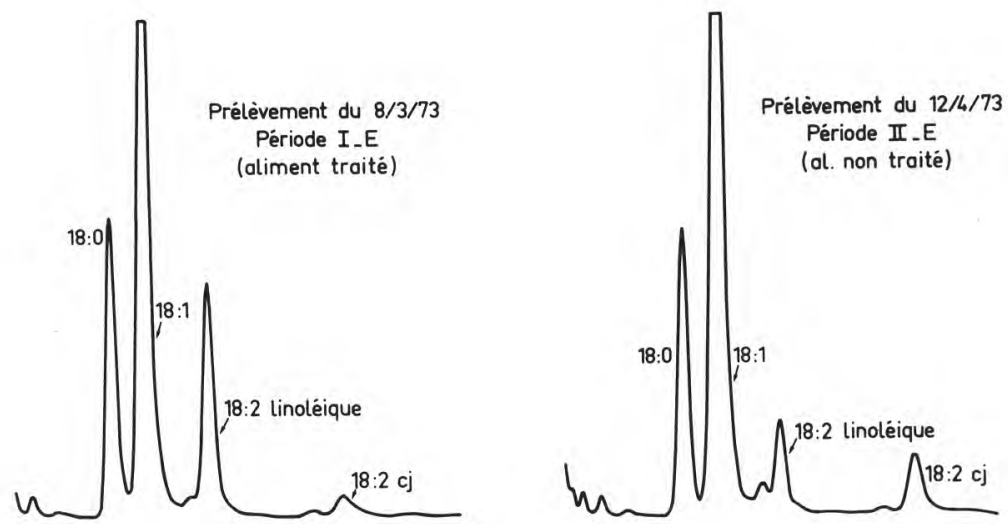

fig. 6

Modification des proportions relatives des acides diènes à 18 atomes de carbone (acide linoléique et acide diène conjugué) à la suite de la consommation par les vaches laitières de l'aliment traité (huile protégée) ou de l'aliment non traité (huile non protégée).
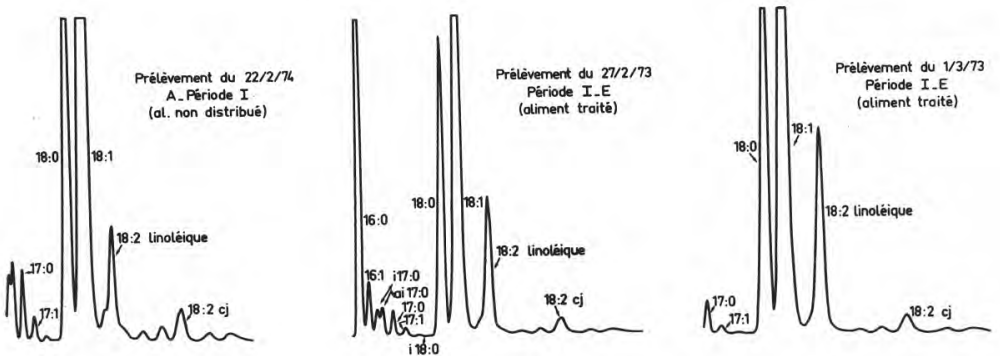

fig. 7

Variation du rapport acide conjugué octadécadiènoïque/acide linoléique selon les conditions de distribution de l'aliment spécial.

impairs comptant 13,15 et 17 atomes de carbone accusent tous une diminution pendant la période de distribution de l'aliment riche en acides poly-insaturés, que cet aliment soit traité ou non, qu'il n'y ait donc pas d'hydrogénation ou qu'il y en ait.

\section{IV. - DISCUSSION}

\section{1) Accroissement du taux d'acide linoléique dans le lait}

L'accroissement du taux d'acide linoléique dans le lait s'est révélé conforme à ce qui avait été souhaité. Les vaches ont consommé sans difficulté le complément alimentaire parfois mêlé au tourteau et distribué pendant la traite. Cependant le rendement de l'opération 
est modeste. Le taux de transfert de l'acide linoléique du supplément alimentaire au lait est seulement de 16 à 17 p. 100. Si un taux de transfert du même ordre (20-22 p. 100) a été signalé par Bitman et al. (1972), les auteurs australiens signalent des taux de transfert supérieurs atteignant même 40 p. 100 (Cook et Scott, 1972).

Du point de vue pratique, l'utilisation d'un complément alimentaire dans lequel l'huile insaturée est "protégée "s'avère un procédé relativement simple pour accroître le taux de l'acide linoléique dans le lait de vache.

Il convient d'estimer le prix de revient du procédé, de le comparer au prix de revient des autres procédés technologiques qui peuvent conduire à des résultats équivalents (fractionnement de la matière grasse laitière par cristallisation, addition directe d'huile végétale, etc.).

\section{2) Les acides mineurs à 18 atomes de carbone formés au cours de I'hydrogénation}

Nous avons établi antérieurement (Kuzdzal-Savoie, 1964 ; KuzdzalSavoie et al., 1966), que l'hydrogénation portant sur l'acide linolénique s'accompagnait également de la formation d'acide diène conjugué à 18 atomes de carbone. Or s'il est bien établi (Kepler et al., 1966 cités par Dawson et Kemp, 1970 ; Kemp et Dawson, 1967) que l'acide cis, trans-octadéca-9,11-diénoïque est formé au cours de l'hydrogénation portant sur l'acide linoléique, il y aurait formation, comme le rappellent Dawson et Kemp (1970), à partir de l'acide linolénique, d'un acide octadécatriénoïque comportant un système conjugué : cis, trans, cisoctadéca-9,11,15-triénoïque. Ultérieurement cet acide serait hydrogéné au niveau de la liaison cis- 9 et conduirait préférentiellement à l'acide trans, cis-octadéca-11,15-diénoïque. Il nous paraît possible que simultanément à la formation de l'acide trans, cis-octadéca-11,15-diénoïque se forme l'acide cis, trans-octadéca-9,11-diénoïque. Ceci permettrait d'expliquer la formation d'acide diène conjugué à partir de l'acide linolénique comme à partir de l'acide linoléique.

Malheureusement, dans l'étude récente consacrée aux aspects quantitatifs de la biohydrogénation des acides gras, Bickerstaffe et al. (1972) n'apportent pas d'information sur ce point particulier.

Nous avons par ailleurs constaté qu'une hydrogénation prononcée (comme il en existe une pour le groupe $\mathrm{E}$ en seconde période de distribution de l'aliment spécial non traité) s'accompagne d'un accroissement notable du pic adjacent, et antérieur au pic de l'acide linoléique. Récemment, Parodi (1973), a indiqué que l'acide trans-16octadécénoïque correspondait à ce pic. Depuis longtemps identifié dans le beurre (Backderf et Brown, 1958) ce pic est également accru lorsque l'hydrogénation porte sur l'acide linolénique (Kuzdzal-Savoie et al., 1966). 


\section{3) Etudes complémentaires}

L'adjonction à la ration alimentaire des vaches laitières du supplément riche en huile de tournesol modifie vraisemblablement la flore du rumen.

Des modifications surviennent dans la composition des lipides sanguins (Cook et Scott, 1972) et se répercutent au niveau de la glande mammaire lors de la synthèse in situ des glycérides.

Enfin la matière grasse du lait plus riche en acide linoléique est en même temps plus vulnérable, c'est-à-dire plus susceptible à l'oxydation génératrice de défauts organoleptiques.

Les résultats obtenus au cours de l'expérimentation et concernant les deux derniers points évoqués seront rassemblés dans une publication ultérieure.

\section{Remerciements}

Nous remercions, en premier lieu, M. Zelter qui a bien voulu s'intéresser à notre travail et se charger, avec le concours de MM. Tissier et Melcion du Laboratoire de Technologie des Aliments des Animaux, de l'opération de tannage de l'aliment.

Nous remercions également $M$. Maubois, Directeur du Laboratoire de Technologie Laitière à Rennes et $\dot{M}$. Le Douaron qui en collaboration avec la Société Bridel ont préparé l'aliment spécial.

Nous remercions, enfin, $M$. Siméon, Directeur du Domaine Expérimental de Bressonvilliers et $M$. Hesnault responsable du déroulement pratique de l'expérimentation.

\section{Rés u mé}

L'objectif était d'obtenir un taux accru d'acide linoléique dans le lait de vache (10 p. 100 au moins des acides gras totaux) par une modification limitée de la ration alimentaire.

Un aliment spécial a été préparé : $112 \mathrm{~kg}$ d'huile de tournesol (62 p. 100 d'acide linoléique) additionnée de $0,5 \mathrm{~g}$ d'acétate d' $\alpha$-tocophérol par $\mathrm{kg}$ d'huile, ont été incorporés à $750 \mathrm{~kg}$ de lait écrémé concentré à 54 p. 100 . Le mélange a été atomisé dans un appareil Spray. La moitié de la poudre obtenue a été agglomérée en petits cylindres de $2 \times 0,5 \mathrm{~cm}$, l'autre moitié a subi un traitement de tannage (brevet déposé les 3 et 8 février 1965 en France) permettant le durcissement de l'enveloppe protidique des grains de poudre et protégeant ainsi la matière grasse vis-à-vis de l'hydrogénation dans le rumen.

L'aliment spécial tanné ou seulement aggloméré a été distribué à raison de $1,2 \mathrm{~kg}$ par jour pendant 1 semaine, de $2,4 \mathrm{~kg}$ pendant 2 semaines et de $1,2 \mathrm{~kg}$ pendant la $4^{\mathrm{e}}$ semaine.

Les résultats de l'analyse des acides gras du lait montrent qu'une distribution de $2,4 \mathrm{~kg}$ par jour d'aliment spécial (E) tanné peut 
accroître le taux d'acide linoléique de la matière grasse du lait de 1,5-2 p. 100 à $11,5-12$ p. 100 des acides gras totaux pour une production journalière d'environ 151 de lait.

Les variations des proportions relatives des divers autres acides (acides monoènes, diènes conjugués, ramifiés) font l'objet d'une discussion.

\section{S u m mar y}

\section{INCREASE IN THE LEVEL OF LINOLEIC ACID IN COW'S MILK}

The purpose of the present study was to increase the level of linoleic acid in cow's milk (at least 10 p. 100 of total fatty acids) by a slight modification of the diet.

A special feed was prepared : $112 \mathrm{~kg}$ sunflower oil (62 p. 100 of linoleic acid) together with $0.5 \mathrm{~g}$ of tocopherol per $\mathrm{kg}$ oil were incorporated into $750 \mathrm{~kg}$ skim milk concentrate (54 p. 100). The mixture was atomized in spray dryer. Half of the powder obtained was transformed into pellets sized $2 \times 0,5 \mathrm{~cm}$, the other half was subjected to a tanning treatments (according to a technique patended in France on February 3rd and 8th 1965 resulting in a hardening of the protein coat of the powder particles, thus protecting the fat against hydrogenation in the rumen.

The special tanned or pelleted diet was offered in the proportion of $1.2 \mathrm{~kg}$ per day during one week, $2.4 \mathrm{~kg}$ per day during 2 weeks and $1.2 \mathrm{~kg}$ per day during the fourth week.

The results of the analysis of milk fatty acids show that the administration of $2.4 \mathrm{~kg}$ per day of the special tanned diet (E) increased the linoleic acid level of the milk fat from 1.5-2 p. 100 to $11.5-12$ p. 100 of total fatty acids for a daily production of about 151 of milk.

Variations in the relative proportions of the other acids (saturated, monoenes, conjugated dienes, brauched fatty acids) form the subject of a discussion.

Reçu pour publication en octobre 1974.

\section{Références bibliographiques}

BACKDERF (H. R.), BRown (J. B.) (1958). - Further contributions to the nature of the monoethenoic fatty acids of butter fat. Arch. Biochem. Biophys., 76, 15-27.

Bickerstaffe (R.), NoAKes (O. E.), ANnison (E. F.) (19..). - Quantitative aspects of fatty acid biohydrogenation, absorption and transfer into milk fat in the lactating goat, with special reference to the cis and trans-isomers of octadecenoate and linoleate. Biochem. J., 130, 607-617. 
Bitman (J.), Dryden (L. P.), Goering (H. K.), Wrenn (T. R.), Yoncoskie (R. A.), EdMONDSON (L. F.) (1973). - Efficiency of transfer of polyunsaturated fats into milk. J. Amer. Oil Chem. Soc., 50, 93-98.

Соок (J.), Scotт (T. W.) (1972). - Formaldehyde-treated casein safflower oil supplement for dairy cows. II. Effect on the fatty acid composition of plasma and milk lipids. J. Dairy Res., 39, 211-218.

Dawson (R. M. C.), KeMP (P.) (1969). - Biohydrogenation of dietary fats in ruminants in physiology of digestion and metabolism in the ruminant. Phillipson A. T. Ed., Ariel Press, Cambridge, 504.

Holman (R. T.) (1968), - Essential fatty acid deficiency. Progress in the chemistry of fats and other lipids. IX (2), 279,348. Pergamon Press, London.

Kemp (P.), Dawson (R. M. C.) (1967), - IIth Int. Congr. Biochem. Problems of Lipids, Jerusalem.

KePler (C. R.), Hirons (K. P.), Mc NeIll (J. J.), Tore (S. B.) (1966). - J. Biol. Chem., 241, 1350. Cité par Dawson (R. M. C.) et Kemp (P.).

Kristensen (V. F.), Andersen (P. E.), Jensen 'G. K.), Hansen (P.S.), Fisker (A. N.), WORTENSEN (B. K.) (1974). - Influence on milk yield, fatty acid composition of milk fat and quality of milk and butter by feeding dairy cows encapsulated soybean oil. Faellesudvalget for Statens Mejeri-og Husdyrbrugsforsog, Hiller $\varnothing \mathrm{d}$ (Danemark). Premier rapport.

Kuzdzal-Savoie (S.) (1964). - Influence de la composition de la ration sur la composition chimique du beurre de vache. Thèse, série $A n^{\circ}$ 4277-5128. Faculté des Sciences de Paris.

KuzDzal-Savoie (S.), Kuzizal (W.) (1961). - Les acides poly-insaturés du beurre. Influence de la saison. Ann. Tech. Agr., 10, 73-80.

Kuzdzal-Savoie (S.), Raymond (J.), KuzDzal (W.), Petit (J.) (1966), — Les acides gras trans du beurre. II. Contribution à l'étude de leur origine. Ann. Biol. anim. Bioch. Biophys., 6, 351-371.

KuzDzal-Savoie (S.), Kuzdzal (W.), Langlois (D.) (1969), - Contribution à l'étude des acides gras diènes du beurre. Fette Seijen Anstrich., 71, 326-330.

Le Breton (E.), Ferret (S.) (1959). - Quelques aspects actuels du problème des acides gras indispensables et essentiels. La notion des familles d'acides polyinsaturés et ses conséquences. Expos. ann. Biochim. méd., 21, 135.

Leroy (F.), Zelter (S.Z.), FrançoIs (A.C.) (1964). - Protection des protéines alimentaires contre la désamination bactérienne au niveau du rumen. Etudes en rumen artificiel. C. R. Acad. Sci., Paris, 259, 1592-1595.

Parod (P. W.) (1973). - The position of trans-octadec-16-enoic acid in the fatty acid profile of milkfat. Anstr. J. Dairy Technol., 28, 161-164.

Plowman (R. D.), Bitman (T.), Gordon (C. H.), Dryden (L. P.), Goering (H. K.), WrenN (T. R.), EDMONDSON (L. F.), YoncoskIE (R. A.), Douglas (F. W.) (1971). Milk fat with increased polyunsaturated fatty acids. J. Dairy Sci., 55, 204-207.

Scott (T. W.), Cook (L. J.), Ferguson (K. A.), Mc Donald (I. W.), Buchanan (R. A.), LofTus Hills (G.) (1970). - Production of polyunsaturated milk fat in domestic ruminants. Anst. J. Sci., 32, 291.

Soderhjelm (L.), Wiese (H. F.), Holman (R. T.) (1970). - The role of polyunsaturated acids in human nutrition and metabolism. Progress in the chemistry of fats and other lipids. IX (4), 555-585. Pergamon Press, London.

Storry (J. E.), Rook (J.A. F.), HaLl (A. J.) (1967). - The effect of the amount and type of dietary fat on milk fat secretion in the cow. Br. J. Nutr., 21, 425.

Zelter (S. Z.), Leroy (F.), Tissier (J. P.) (1970). - Protection des protéines alimentaires contre la désamination bactérienne dans le rumen. I. Etudes in vitro: comportement en milieu de rumen de quelques protéines tannées avec du tanin de châtaignier ou certains aldéhydes (formaldéhyde, glutaraldéhyde, glyoxal). Ann. Biol. anim. Bioch. Biophys., 10, 111-122. 